\title{
Effect of Pelvic Lymphadenectomy on Survival in Patients with Low-Risk Early-Stage Endometrial Cancer Diagnosed Intraoperatively Using Frozen Tissue Sections: A Retrospective Analysis
}

This article was published in the following Dove Press journal:

Cancer Management and Research

\author{
Caiyan Liu' \\ Jianguo Zhao' \\ Shasha Liu' \\ Yaomei $\mathrm{Ma}^{2}$ \\ Yun Yang ${ }^{3}$ \\ Pengpeng $\mathrm{Qu}{ }^{\prime}$ \\ 'Department of Gynecological Oncology, \\ Tianjin Central Hospital of Gynecology \\ Obstetrics, Tianjin, People's Republic of \\ China; ${ }^{2}$ Department of Gynecological \\ Oncology, Tianjin Medical University \\ Cancer Institute \& Hospital, Tianjin, \\ People's Republic of China; ${ }^{3}$ Department \\ Of Gynecology, Tianjin Central Hospital \\ of Gynecology Obstetrics, Tianjin, \\ People's Republic of China
}

Correspondence: Pengpeng Qu Department of Gynecological Oncology, Tianjin Central Hospital of Gynecology Obstetrics, Tianjin, People's Republic of China

Tel +86-18920196050

Email qu.pengpeng@hotmail.com
Purpose: To determine whether pelvic lymphadenectomy improved survival in patients diagnosed with low-risk early-stage endometrial cancer by intraoperative pathology.

Methods: This retrospective analysis included 238 patients at our hospital.

Results: The lymphadenectomy and non-lymphadenectomy groups contained 121 and 117 patients, respectively. In both groups, more than half the patients had tumor size $\geq 2 \mathrm{~cm}$, and most had myometrial invasion $<50 \%$, stage Ia disease and no lymphovascular space invasion. Age, tumor size, myometrial invasion, surgical-pathologic stage and postoperative adjuvant therapy use were comparable between groups. The non-lymphadenectomy group had more patients treated laparoscopically $(36.8 \%$ vs $10.7 \% ; P<0.001)$ and fewer patients with histologic grade 2 disease $(35.9 \%$ vs $62.8 \% ; P<0.001)$ than the lymphadenectomy group. In the non-lymphadenectomy group, intraoperative frozen section pathology disagreed with postoperative pathology in only $31 / 117$ cases for histologic grade (none upgraded to grade 3 ), 1 / 117 cases for myometrial invasion (one case revised from $<50 \%$ to $\geq 50 \%$ ) and $3 / 117$ cases for surgical-pathologic stage (upgraded from Ia to Ib or II). Disease recurrence rate and overall survival did not differ significantly between the lymphadenectomy and nonlymphadenectomy groups. In multivariate Cox regression analysis, only surgical-pathologic stage $>$ Ia (odds ratio, 47.7; 95\% confidence interval, 6.7-340.8; $P=0.031$ ) was associated with increased odds of disease recurrence.

Conclusion: Pelvic lymphadenectomy may not be necessary in patients with an intraoperative diagnosis of low-risk endometrial cancer.

Keywords: endometrial cancer, lymph node excision, prognosis, recurrence, survival

\section{Introduction}

Endometrial cancer is the most common gynecologic malignancy worldwide, accounting for $5 \%$ of all cancers and $2 \%$ of cancer-related deaths in women. ${ }^{1}$ Management strategies for endometrial cancer include hysterectomy with bilateral salpingo-oophorectomy, hormonal therapy, radiotherapy and chemotherapy, and the overall survival rate is nearly $90 \%$ for early-stage disease. ${ }^{2}$

Previous investigations have defined low-risk early-stage endometrial cancer as disease of endometrioid histology and histologic grade $1 / 2$ confined to the uterine corpus with $<50 \%$ myometrial invasion. ${ }^{3,4}$ The Mayo algorithm includes tumor diameter $\leq 2 \mathrm{~cm}$ as an additional criterion for low-risk endometrial cancer. ${ }^{5}$ Patients 
with low-risk early-stage endometrial cancer have a very low lymph node metastasis rate, especially for tumors $\leq 2 \mathrm{~cm}$ in diameter, and a 5 -year survival rate of $96 \% .{ }^{4-6}$ The clinical benefits of retroperitoneal lymphadenectomy during surgical treatment of low-risk early-stage endometrial cancer remain unclear. ${ }^{5}$ Previous studies reported that pelvic lymphadenectomy did not improve recurrence-free or overall survival in patients with stage I endometrial cancer ${ }^{7-10}$ but was associated with increased costs and a higher risk of complications in patients with low-risk endometrial cancer. ${ }^{11,12}$ Omitting lymphadenectomy in patients with low-risk endometrial cancer requires an accurate diagnosis before surgery is completed. However, most studies investigating the relevance of lymphadenectomy have relied on postoperative assessments to confirm the presence of low-risk early-stage endometrial cancer. Few studies have used intraoperative microscopy to evaluate fresh frozen tissue samples. ${ }^{13}$

We hypothesized that intraoperative frozen section pathology could identify patients with low-risk early-stage endometrial cancer and that adding lymphadenectomy to hysterectomy with bilateral salpingo-oophorectomy would have no clinical benefit in this patient group. Therefore, this study aimed to compare intraoperative frozen section pathology with postoperative pathology findings and determine whether pelvic lymphadenectomy influenced disease recurrence and overall survival in patients with low-risk earlystage endometrial cancer.

\section{Patients and Methods Study Design and Participants}

This retrospective analysis included patients with stage I endometrial cancer treated at our hospital between April 2012 and September 2015. The inclusion criteria were: 1) endometrioid adenocarcinoma (grade 1/2) confirmed by preoperative diagnostic curettage; 2) preoperative magnetic resonance imaging (MRI) showed the tumor was limited to the uterine body with no suspected pelvic metastasis or enlarged retroperitoneal lymph nodes; 3) intraoperative frozen section pathology indicated histologic grade $1 / 2$ and myometrial invasion $<50 \%$; and 4 ) cervical metastasis or extrauterine lesions were not visible during surgery. Tumor size was not an entry criterion. The exclusion criteria were: 1) preoperative radiotherapy, chemotherapy and/or hormone therapy; 2) non-endometrioid adenocarcinoma; 3) histologic grade 3; 4) other systemic malignancies; and 5) intraoperative frozen section pathology not meeting the criteria for low-risk endometrial cancer. The study was approved by the ethics committee of the Tianjin Central Hospital of Gynecology Obstetrics. The waiver of patient's informed consent was approved by the ethics committee because this was a retrospective study in which management was not intervened and prognosis of patients was not influenced. The study was performed following the Declaration of Helsinki and all following amendments. The authors guarantee the privacy of patients and the confidentiality of the patient data.

\section{Surgery}

All patients underwent total abdominal or laparoscopic hysterectomy with bilateral salpingo-oophorectomy. Some patients also underwent pelvic lymphadenectomy according to the results of intra-operative pelvic examination, intraoperative frozen section pathology or at the discretion of the attending surgeon. The operations were performed by a senior deputy chief physician and chief physician with extensive experience.

\section{Postoperative Adjuvant Therapy}

In accordance with the 2009 FIGO guidelines, ${ }^{14}$ postoperative radiotherapy and/or chemotherapy was performed in patients with high-risk factors, including age $>60$ years or postoperative pathology findings of deep myometrial invasion, histologic grade 3, lymphovascular space invasion, cervical interstitial infiltration or extrauterine metastasis.

\section{Follow-Up}

Patients were followed up postoperatively every 3 months for the first 2 years, every 6 months for the subsequent 3 years and then annually. Follow-up included gynecologic examination, measurement of serum tumor markers, chest X-ray, and pelvic color Doppler ultrasound or MRI. Disease recurrence was defined as physical or imaging evidence of tumor reappearance in the pelvic cavity or other body regions after treatment.

\section{Clinical Data Collection}

Information extracted from the medical records included age, surgical method, tumor diameter, myometrial invasion depth, histologic grade, lymphovascular space invasion status, number of lymph nodes resected (lymphadenectomy group), surgical-pathologic stage, postoperative adjuvant therapy, follow-up duration, disease recurrence during follow-up and death during follow-up. 


\section{Statistical Analysis}

The analysis was performed using SPSS 20.0 (IBM Corp., Armonk, NY, USA). Normally distributed continuous data are described as mean \pm standard deviation and were compared using the independent-samples $t$-test. Non-normally distributed continuous data are presented as median (range) and were compared using the Mann-Whitney $U$-test. Count data are presented as $\mathrm{n}(\%)$ and were compared using the chi-squared test or Fisher's exact test (expected frequency of $>20 \%$ of the cells was $<5$ ). Survival analysis was performed using the Kaplan-Meier method and Log-rank test. Univariate and multivariate Cox regression analyses were used to identify factors associated with disease recurrence, with calculation of odds ratios and $95 \%$ confidence intervals. Significant factors $(P<0.05)$ in the univariate analysis were entered into the multivariate analysis. A two-sided $P$-value $<0.05$ was considered statistically significant.

\section{Results}

\section{Baseline Clinical Characteristics}

A total of 238 patients were included in the analysis, with 40 patients $(16.8 \%)$ aged $\geq 60$ years. Pelvic lymphadenectomy was performed in 121 patients (50.8\%), and among these patients, 32 (26.4\%) had $\geq 20$ lymph nodes resected, 64 (52.9\%) had 21-30 lymph nodes resected, and 25 (20.7\%) had $\geq 31$ lymph nodes resected. The baseline clinical characteristics are shown in Table 1. In both groups, more than half the patients had a tumor size $\geq 2 \mathrm{~cm}$, and most had myometrial invasion $<50 \%$, stage Ia disease, no lymphovascular space invasion and no postoperative adjuvant therapy. There were no significant differences between groups in age, tumor size, myometrial invasion depth, disease stage or use of postoperative radiotherapy or chemotherapy. Laparoscopic surgery was used more frequently in the non-lymphadenectomy group than in the lymphadenectomy group $(36.8 \%$ vs $10.7 \% ; P<0.001)$. Histologic grade 2 disease was more common in the lymphadenectomy group than in the non-lymphadenectomy group $(62.8 \%$ vs $35.9 \% ; P<0.001)$.

\section{Comparison of Findings Between Intraoperative Frozen Section Pathology and Postoperative Pathology for the Non-Lymphadenectomy Group}

Intraoperative frozen section pathology suggested complex atypical hyperplasia, grade 1 disease and grade 2 disease in 10, 86 and 21 patients, respectively. Postoperative pathology altered the diagnosis in $9 / 10$ cases diagnosed intraoperatively as complex atypical hyperplasia, 21/86 cases diagnosed as grade 1 , and $1 / 21$ cases diagnosed as grade 2 . One case suggested complex atypical hyperplasia in postoperative pathology without residual tumor (Table 2). No cases were upgraded to histologic grade 3 by postoperative pathology. Intraoperative frozen section pathology suggested no intramuscular infiltration (complex atypical hyperplasia) in 10/ 117 patients and myometrial invasion $<50 \%$ in all remaining patients; only 1 patient was subsequently confirmed to have myometrial invasion $\geq 50 \%$ by postoperative pathology. All patients in the non-lymphadenectomy group had surgicalpathologic stage Ia disease according to intraoperative frozen section pathology, and postoperative pathology upgraded this to stage Ib in 1 patient and stage II in 2 patients.

\section{Factors Associated with Disease Recurrence}

Patients in the non-lymphadenectomy group were followed up for a median 47 months (range, 0-79 months; interquartile interval, 40-58 months), and disease recurrence occurred in 2 patients $(1.7 \%)$. In the first case, the original tumor was $10 \mathrm{~mm}$ in diameter, histologic grade 2 and limited to the uterine isthmus without invasion of cervical mucosa or stroma. Recurrence (as a vaginal mass) was diagnosed 2 years later. The patient was treated with chemotherapy (paclitaxel/carboplatin) and radiotherapy, and no further recurrence occurred during the subsequent 18-month follow-up. In the second case (tumor diameter, $22 \mathrm{~mm}$; histologic grade 1; myometrial invasion $\geq 50 \%$; lymphovascular space invasion), the patient received no postoperative adjuvant therapy and presented $>2$ years later with a pelvic mass and serum CA125 of 102 $\mathrm{mIU} / \mathrm{mL}$. The patient was treated with chemotherapy (paclitaxel/carboplatin) and radiotherapy. There was no evidence of recurrence during the subsequent 20-month follow-up.

Patients in the lymphadenectomy group were followed up for a median 48 months (range, 2-83 months; interquartile interval, 38-67 months), and disease recurrence occurred in 2 patients $(1.7 \%$ ). In the first case (tumor diameter, $25 \mathrm{~mm}$; histologic grade 2; myometrial invasion $<50 \%$ ), brain metastasis was diagnosed 25 months after surgery, and the patient died after 6 months of palliative treatment. In the second case (tumor diameter $<2 \mathrm{~cm}$; histologic grade 2; myometrial invasion $<50 \%$ ), postoperative pathology showed left ovarian metastatic carcinoma 
Table I Baseline Clinical Characteristics of the Study Participants

\begin{tabular}{|c|c|c|c|c|}
\hline & $\begin{array}{l}\text { Non-Lymphadenectomy Group } \\
(\mathrm{N}=1 \mid 7)\end{array}$ & $\begin{array}{l}\text { Lymphadenectomy Group } \\
(N=12 I)\end{array}$ & $\begin{array}{l}\text { Total } \\
(\mathbf{N}=238)\end{array}$ & $\boldsymbol{P}$ \\
\hline Age & & & & 0.418 \\
\hline$<60$ years & $95(81.2 \%)$ & $103(85.1 \%)$ & $198(83.2 \%)$ & \\
\hline$\geq 60$ years & $22(18.8 \%)$ & $18(14.9 \%)$ & $40(16.8 \%)$ & \\
\hline Surgical method & & & & $<0.001$ \\
\hline Total abdominal hysterectomy & $74(63.2 \%)$ & $108(89.3 \%)$ & $182(76.5 \%)$ & \\
\hline Total laparoscopic hysterectomy & $43(36.8 \%)$ & $13(10.7 \%)$ & $56(23.5 \%)$ & \\
\hline Tumor size & & & & 0.072 \\
\hline$<2 \mathrm{~cm}$ & $56(47.9 \%)$ & $44(36.4 \%)$ & $100(42.0 \%)$ & \\
\hline$\geq 2 \mathrm{~cm}$ & $61(52.1 \%)$ & 77 (63.6\%) & $138(58.0 \%)$ & \\
\hline Myometrial invasion $\S$ & & & & 1.000 \\
\hline$<50 \%$ & 115 (99.1\%) & $119(99.2 \%)$ & $234(99.2 \%)$ & \\
\hline$\geq 50 \%$ & $\mathrm{I}(0.9 \%)$ & $\mathrm{I}(0.8 \%)$ & $2(0.8 \%)$ & \\
\hline Histologic grade ${ }^{\S}$ & & & & $<0.001$ \\
\hline Complex atypical hyperplasia & I (0.9\%) & $\mathrm{I}(0.8 \%)$ & $2(0.8 \%)$ & \\
\hline 1 & $74(63.2 \%)$ & $44(36.4 \%)$ & $118(49.6 \%)$ & \\
\hline 2 & $42(35.9 \%)$ & $76(62.8 \%)$ & $118(49.6 \%)$ & \\
\hline Lymphovascular space invasion & & & & 0.343 \\
\hline Negative & $103(88 \%)$ & III (9I.7\%) & $214(89.9 \%)$ & \\
\hline Positive & 14 (I2\%) & $10(8.3 \%)$ & $24(10.1 \%)$ & \\
\hline Surgical-pathologic stage $\S$ & & & & 0.903 \\
\hline la & II (97.4\%) & II 8 (97.5\%) & $232(97.5 \%)$ & \\
\hline $\mathrm{lb}$ & $\mathrm{I}(0.9 \%)$ & $\mathrm{I}(0.8 \%)$ & $2(0.8 \%)$ & \\
\hline II & $2(1.7 \%)$ & I (0.8\%) & $3(1.3 \%)$ & \\
\hline Illb & $0(0.0 \%)$ & I (0.8\%) & I (0.4\%) & \\
\hline Postoperative treatment & & & & 1.000 \\
\hline Radiotherapy & 15 (12.8\%) & $16(13.2 \%)$ & 31 (13.0\%) & \\
\hline Chemoradiotherapy & $0(0.0 \%)$ & $\mathrm{I}(0.8 \%)$ & $\mathrm{I}(0.8 \%)$ & \\
\hline None & $102(87.2 \%)$ & 104 (86.0\%) & $206(86.6 \%)$ & \\
\hline
\end{tabular}

Note: ${ }^{\S}$ Based on postoperative pathology.

Table 2 Comparison of Histologic Grade Between Intraoperative Frozen Section Pathology and Postoperative Pathology for Patients in the Non-Lymphadenectomy Group

\begin{tabular}{|l|l|l|l|l|l|l|}
\hline \multirow{2}{*}{ Intraoperative Pathology } & \multicolumn{2}{|l|}{ Postoperative Pathology } & & \multirow{2}{*}{ Sensitivity (\%) } & \multirow{2}{*}{ Specificity (\%) } \\
\cline { 2 - 6 } & \multicolumn{2}{|l|}{ Complex Atypical Hyperplasia } & Grade I & Grade 2 & Total & \\
\hline Complex atypical hyperplasia & $\mathrm{I}$ & 8 & $\mathrm{I}$ & 10 & 100.0 & 92.2 \\
Grade I & 0 & 65 & 21 & 86 & 87.8 & 51.2 \\
Grade 2 & 0 & 1 & 20 & 21 & 47.6 & 98.7 \\
Total & $\mathrm{I}$ & 74 & 42 & 117 & & \\
\hline
\end{tabular}

(stage IIIb). The patient was treated with radiotherapy and chemotherapy (paclitaxel/carboplatin), but pelvic recurrence and lung metastasis occurred 16 months after treatment discontinuation, and the patient died 10 months later.
Univariate Cox regression analysis indicated that myometrial invasion $\geq 50 \%$ (odds ratio, 37.3 ; $95 \%$ confidence interval, 3.9-358.9; $P=0.002$ ), lymphovascular space invasion (odds ratio, 8.6; 95\% confidence interval, 1.2-61.1; 
$P=0.031$ ) and surgical-pathologic stage $>$ Ia (odds ratio, 47.7; 95\% confidence interval, 6.7 $-340.8 ; P=0.031$ ) were associated with increased odds of postoperative disease recurrence. However, age, surgical method, tumor size, histologic grade, postoperative adjuvant therapy and pelvic lymphadenectomy were not associated with postoperative disease recurrence (Table 3 ). In addition to significant factors $(P<0.05)$ in the univariate analysis, pelvic lymphadenectomy was also entered into the multivariate analysis because it was the focus of this study. Multivariate analysis revealed that surgical-pathologic stage $>$ Ia (odds ratio, 47.7; 95\% confidence interval, 6.7-340.8; $P=0.031$ ) was the only factor associated with increased likelihood of recurrence (Table 3).

\section{Survival Analysis}

No patients in the non-lymphadenectomy group died, whereas both patients with disease recurrence in the lymphadenectomy group died (1.7\%). Overall survival did not differ significantly between the two groups (Figure 1A). However, poorer survival was observed for patients with lymphovascular space invasion $(P=0.010$; Figure $1 \mathrm{~B})$ or stage $>1$ a disease $(P<0.001$; Figure $1 C)$.

\section{Discussion}

A notable finding of this study was that intraoperative frozen section pathology showed acceptable accuracy in the diagnosis of endometrial cancer, with no cases upgraded from histologic grade 1 or 2 to grade 3 and

Table 3 Cox Regression Analysis of Factors Associated with Disease Recurrence

\begin{tabular}{|c|c|c|c|c|c|c|}
\hline & \multicolumn{3}{|c|}{ Univariate Analysis } & \multicolumn{3}{|c|}{ Multivariate Analysis } \\
\hline & Odds Ratio & 95\% Confidence Interval & $\boldsymbol{P}$ & Odds Ratio & 95\% Confidence Interval & $\mathbf{P}$ \\
\hline $\begin{array}{l}\text { Age } \\
\qquad 60 \text { years } \\
\quad \geq 60 \text { years }\end{array}$ & $\begin{array}{l}\text { Ref. } \\
4.85\end{array}$ & $0.68-34.4 I$ & 0.115 & & & \\
\hline $\begin{array}{l}\text { Surgical method } \\
\text { Total abdominal hysterectomy } \\
\text { Total laparoscopic hysterectomy }\end{array}$ & $\begin{array}{l}\text { Ref. } \\
0.03\end{array}$ & $0.00-721.26$ & 0.504 & & & \\
\hline $\begin{array}{l}\text { Tumor size } \\
\qquad 2 \mathrm{~cm} \\
\geq 2 \mathrm{~cm}\end{array}$ & $\begin{array}{l}\text { Ref. } \\
0.711\end{array}$ & $0.10-5.05$ & 0.733 & 0.45 & $0.04-5.77$ & 0.542 \\
\hline $\begin{array}{l}\text { Myometrial invasion } \\
\qquad 50 \% \\
\geq 50 \%\end{array}$ & $\begin{array}{l}\text { Ref. } \\
37.33\end{array}$ & $3.88-358.87$ & 0.002 & 1.02 & $0.03-41.12$ & 0.991 \\
\hline $\begin{array}{l}\text { Histologic grade } \\
\qquad \begin{array}{l}1 \\
2\end{array}\end{array}$ & $\begin{array}{l}\text { Ref. } \\
2.91\end{array}$ & $0.30-27.97$ & 0.355 & & & \\
\hline $\begin{array}{l}\text { Lymphovascular space invasion } \\
\text { Negative } \\
\text { Positive }\end{array}$ & $\begin{array}{l}\text { Ref. } \\
8.60\end{array}$ & $1.21-61.06$ & 0.031 & 2.47 & $0.14-42.69$ & 0.534 \\
\hline $\begin{array}{l}\text { Surgical-pathologic stage } \\
\text { la } \\
\text { >la }\end{array}$ & $\begin{array}{l}\text { Ref. } \\
47.70\end{array}$ & $6.68-340.79$ & $<0.001$ & 30.07 & $2.18-4 \mid 4.32$ & 0.011 \\
\hline $\begin{array}{l}\text { Postoperative treatment } \\
\text { None } \\
\text { Chemo/radiotherapy }\end{array}$ & $\begin{array}{l}\text { Ref. } \\
2.14\end{array}$ & $0.22-20.56$ & 0.510 & & & \\
\hline $\begin{array}{l}\text { Pelvic lymphadenectomy } \\
\text { No } \\
\text { Yes }\end{array}$ & $\begin{array}{l}\text { Ref. } \\
0.97\end{array}$ & $0.14-6.92$ & 0.979 & 1.26 & $0.15-10.95$ & 0.832 \\
\hline
\end{tabular}



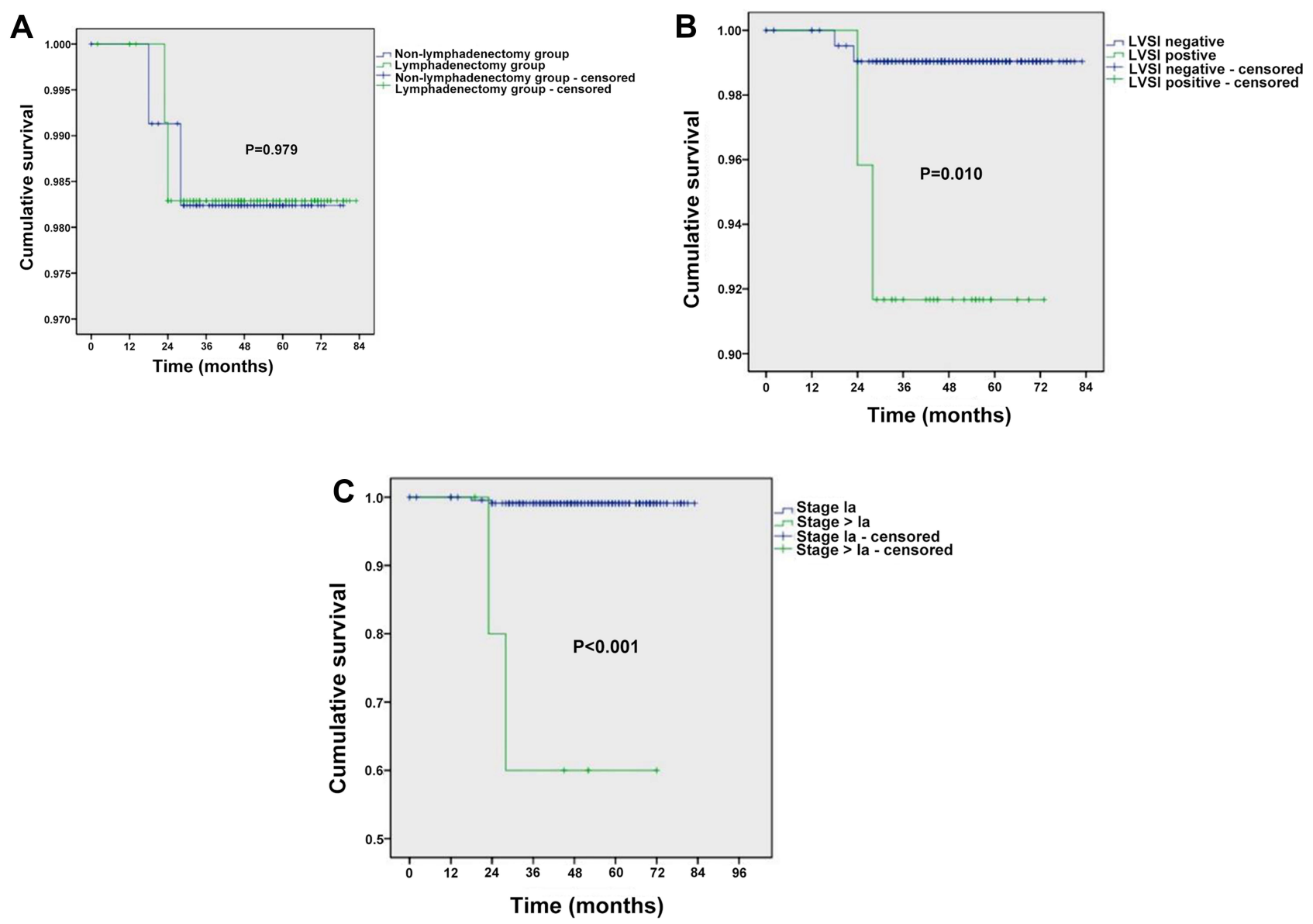

Figure I Kaplan-Meier analysis of overall survival. (A) Stratified analysis based on whether pelvic lymphadenectomy was performed. (B) Stratified analysis based on whether lymphovascular space invasion (LVSI) was present. (C) Stratified analysis based on surgical-pathologic stage (la vs $>$ la). P-values were calculated using the Log-rank test.

only one case upgraded from myometrial invasion $<50 \%$ to invasion $\geq 50 \%$. Importantly, disease recurrence rate and overall survival did not differ significantly between the lymphadenectomy and non-lymphadenectomy groups. Furthermore, multivariate Cox regression analysis identified surgical-pathologic stage $>$ Ia as the only factor associated with increased odds of disease recurrence, with no association between pelvic lymphadenectomy and recurrence. Our findings support the view that pelvic lymphadenectomy may not be necessary in patients with an intraoperative diagnosis of low-risk endometrial cancer.

A major challenge facing gynecologic oncologists is identifying patients who might benefit from lymphadenectomy. The FIGO Cancer Report 2015 recommended excision of enlarged or suspicious lymph nodes in all patients with endometrial cancer, with complete pelvic lymphadenectomy and resection of enlarged para-aortic nodes for high-risk patients. ${ }^{15}$ The 2018 National Comprehensive Cancer Network guideline considered lymph node resection important for comprehensive staging of lesions confined to the uterus but stated that lymphadenectomy might not be appropriate in some patients, although the difficulty identifying patients unsuitable for lymphadenectomy was noted. ${ }^{16}$ According to the Mayo criteria, although selective lymphadenectomy is reasonable, the significance of the stage of lymphadenectomy is greater than the therapeutic value of early-stage patients. Algorithms have been developed in an attempt to predict the risk of lymph node metastasis and identify patients requiring lymphadenectomy. In one study, para-aortic lymph node excision was considered unnecessary in patients classified as low-risk based on serum CA125 level and tumor volume, grade and histology. ${ }^{17}$ Another report utilized preoperative assessments including MRI and serum CA125 level to identify patients with a low risk of lymph node metastasis who did not require lymphadenectomy. ${ }^{18}$ Patients with low-risk early-stage endometrial cancer have a lymph node metastasis rate $<4 \%$, particularly for tumors $\leq 2 \mathrm{~cm}$ in diameter. ${ }^{4-6,19-22}$ The present study defined low-risk endometrial cancer 
with the most commonly used criteria: endometrioid carcinoma with histologic grade 1 or 2 and myometrial invasion $<50 \%$. $^{3,4,23}$ However, some investigations have included tumor diameter $\leq 2 \mathrm{~cm}$ as an additional criterion for low-risk endometrial cancer, ${ }^{5,24}$ and recent studies have suggested optimal tumor size cutoff values of $2.5 \mathrm{~cm}^{25}$ and $3.5 \mathrm{~cm}^{26}$

Univariate Cox regression indicated that myometrial invasion $\geq 50 \%$, lymphovascular space invasion and surgical-pathologic stage $>$ Ia were associated with greater likelihood of disease recurrence, although surgical-pathologic stage $>$ Ia was the only significant factor in multivariate analysis. Notably, tumor size was not associated with disease recurrence. Previously reported risk factors for recurrence of low-risk endometrial cancer include age, surgicalpathologic stage, myometrial invasion $\geq 50 \%$, presence of lymphovascular space invasion, histologic grade 3 , tumor diameter $\geq 2 \mathrm{~cm}$ and postoperative adjuvant therapy. ${ }^{27-31}$ The recurrence rate in the present study was only $1.7 \%$ (4 patients), which may have limited the power of our analysis to identify all factors associated with recurrence. Nevertheless, inspection of the odds ratios suggests that lymphadenectomy was not associated with disease recurrence in our cohort.

Intraoperative frozen section pathology requires good diagnostic accuracy to be used to facilitate decisionmaking regarding whether to perform lymphadenectomy in patients with endometrial cancer. A previous study described high coincidence rates (mostly $>90 \%$ ) between intraoperative frozen section pathology findings and the final pathologic diagnosis, ${ }^{32}$ and the Mayo Clinic reported an inconsistency between methods of only $1.3 \%{ }^{33}$ However, some authors have suggested that an accurate pathologic diagnosis during surgery can only be made by institutions with a high level of expertise. ${ }^{34}$ Intraoperative frozen section pathology has high requirements regarding sample preparation and evaluation, and many hospitals in China may not be in a position to provide an accurate diagnosis using this technique. Indeed, diagnostic agreement rate between intraoperative frozen section pathology and postoperative pathology is $70-80 \%$ in China, ${ }^{35}$ lower than that reported in other countries. All patients in our study were diagnosed with endometrioid carcinoma (grade 1/2) by preoperative diagnostic curettage. In the non-lymphadenectomy group, 10 cases were diagnosed as complex atypical hyperplasia by intraoperative frozen section pathology, but postoperative pathology altered the diagnosis to histologic grade 1 in 8 cases and grade
2 in 1 case. One case suggested complex atypical hyperplasia in postoperative pathology without residual tumor. Importantly, there were no instances of pathologic upgrade to grade 3 . The postoperative pathological upgrade could be attributed to the limited availability of frozen samples, and the most severe lesions were not utilized as specimens. The reason for atypical hyperplasia observed in the intraoperative frozen section and postoperative pathology was that the lesions were scraped out during preoperative diagnosis and no residual tumor was detected. The overall coincidence rate between intraoperative frozen section pathology and postoperative pathology in the diagnosis of myometrial invasion was similar to that reported previously. ${ }^{35}$ The ability of intraoperative frozen section pathology to diagnose myometrial invasion depth depends on the sampling method. For example, a sample permitting accurate diagnosis can be obtained when myometrial invasion is visible to the naked eye during surgery, whereas random sampling when myometrial invasion is not visible might result in missed lesions.

Notably, intraoperative frozen section has limitations in the diagnosis of lymphovascular space invasion. Among the 117 patients who did not undergo lymphadenectomy, postoperative pathology revealed 14 cases of lymphovascular space invasion, 2 cases of cervical interstitial infiltration and 1 case of deep myometrial invasion. Fifteen patients received postoperative adjuvant radiotherapy due to the presence of high-risk factors, and no serious adverse effects were reported. Two patients with disease recurrence were treated with chemoradiotherapy; further recurrence was not observed after treatment, and neither patient died during the study period. Among the 121 patients who underwent pelvic lymphadenectomy, postoperative pathology showed 10 cases of lymphovascular space invasion, 1 case of cervical interstitial infiltration and 1 case of deep myometrial invasion. Fifteen patients were considered to be at high risk and given radiotherapy. Importantly, overall survival did not differ significantly between the lymphadenectomy and nonlymphadenectomy groups. There were 3 cases of postoperative pathologic stage upgrade in each of the nonlymphadenectomy group (to stage Ib in 1 case and stage II in 2 cases) and lymphadenectomy group (to stage Ib, II and $\mathrm{IIIb}$ in 1 case each). After postoperative adjuvant radiotherapy/chemoradiotherapy, only the patient with stage IIIb disease had pelvic recurrence, and this patient subsequently died due to lung metastasis. Survival analysis indicated that advanced surgical-pathologic stage and 
lymphovascular space invasion were related to survival, whereas pelvic lymphadenectomy was not. Previous studies have also concluded that pelvic lymphadenectomy does not improve overall survival in patients with low-risk endometrial cancer. ${ }^{7-10}$ Based on our results and published data, we suggest that pelvic lymphadenectomy may not be indicated in patients diagnosed with low-risk endometrial cancer by intraoperative frozen section pathology.

This study has some limitations. This was not a randomized controlled trial, so the findings should be interpreted with caution. As this was a retrospective analysis, it may be prone to selection and/or information bias. This was a single-center study, so the generalizability of the findings is unknown. The study may have been underpowered to detect some real effects because of the small sample size. The follow-up time was limited. Large-scale, multicenter, randomized controlled trials are needed to confirm our findings.

\section{Conclusion}

Our findings support the proposal that pelvic lymphadenectomy may not be necessary in patients with an intraoperative diagnosis of low-risk endometrial cancer. For patients identified postoperatively as having high-risk factors or disease recurrence, treatment with adjuvant therapy appears to be associated with a good prognosis.

\section{Ethics Approval and Informed Consent}

The study was approved by the Ethics Committee of the Tianjin Central Hospital of Gynecology Obstetrics, Tianjin. The requirement for informed consent was waived due to the retrospective study design. All patients provided informed consent for their treatment.

\section{Author Contributions}

All authors contributed to data analysis, drafting or revising the article, have agreed on the journal to which the article will be submitted, gave final approval of the version to be published, and agree to be accountable for all aspects of the work.

\section{Funding}

There is no funding to report.

\section{Disclosure}

All authors declare that they have no competing interests.

\section{References}

1. Torre LA, Islami F, Siegel RL, Ward EM, Jemal A. Global cancer in women: burden and trends. Cancer Epidemiol Biomarkers Prev. 2017;26(4):444-457. doi:10.1158/1055-9965. EPI-16-0858

2. Makker A, Goel MM. Tumor progression, metastasis, and modulators of epithelial-mesenchymal transition in endometrioid endometrial carcinoma: an update. Endocr Relat Cancer. 2016;23(2):R85-R111. doi:10.1530/ERC-15-0218

3. Morrow CP, Bundy BN, Kurman RJ, et al. Relationship between surgical-pathological risk factors and outcome in clinical stage I and II carcinoma of the endometrium: a gynecologic oncology group study. Gynecol Oncol. 1991;40(1):55-65. doi:10.1016/00908258(91)90086-K

4. Carey MS, O'Connell GJ, Johanson CR, et al. Good outcome associated with a standardized treatment protocol using selective postoperative radiation in patients with clinical stage I adenocarcinoma of the endometrium. Gynecol Oncol. 1995;57(2):138-144. doi:10.1006/ gyno.1995.1115

5. Mariani A, Webb MJ, Keeney GL, Haddock MG, Calori G, Podratz KC. Low-risk corpus cancer: is lymphadenectomy or radiotherapy necessary? Am J Obstet Gynecol. 2000;182(6):1506-1519. doi: $10.1067 / \mathrm{mob} .2000 .107335$

6. Lotocki RJ, Copeland LJ, DePetrillo AD, Muirhead W. Stage I endometrial adenocarcinoma: treatment results in 835 patients. Am J Obstet Gynecol. 1983;146(2):141-145. doi:10.1016/0002-9378(83) 91042-6

7. Benedetti Panici P, Basile S, Maneschi F, et al. Systematic pelvic lymphadenectomy vs. no lymphadenectomy in early-stage endometrial carcinoma: randomized clinical trial. $J$ Natl Cancer Inst. 2008;100(23):1707-1716. doi:10.1093/jnci/djn397

8. Group A, Kitchener H, Swart AM, Qian Q, Amos C, Parmar MK. Efficacy of systematic pelvic lymphadenectomy in endometrial cancer (MRC ASTEC trial): a randomised study. Lancet. 2009;373 (9658):125-136.

9. Turkler C, Kulhan M, Kulhan NG, Ata N, Sanci M, Ozeren M. Role of lymphadenectomy in disease-free and overall survival on low risk endometrium cancer patients. Ginekol Pol. 2018;89(6):311-315. doi:10.5603/GP.a2018.0053

10. Hidaka T, Nakashima A, Shima T, Hasegawa T, Saito S. Systemic lymphadenectomy cannot be recommended for low-risk corpus cancer. Obstet Gynecol Int. 2010;2010:490219. doi:10.1155/2010/ 490219

11. Dowdy SC, Borah BJ, Bakkum-Gamez JN, et al. Prospective assessment of survival, morbidity, and cost associated with lymphadenectomy in low-risk endometrial cancer. Gynecol Oncol. 2012;127 (1):5-10. doi:10.1016/j.ygyno.2012.06.035

12. Frost JA, Webster KE, Bryant A, Morrison J. Lymphadenectomy for the management of endometrial cancer. Cochrane Database Syst Rev. 2017;10:CD007585.

13. Bell JG, Patterson DM, Klima J, Harvison M, Rath K, Reid G. Outcomes of patients with low-risk endometrial cancer surgically staged without lymphadenectomy based on intra-operative evaluation. Gynecol Oncol. 2014;134(3):505-509. doi:10.1016/j.ygyno.2014.06.028

14. Pecorelli S. Revised FIGO staging for carcinoma of the vulva, cervix, and endometrium. Int J Gynaecol Obstet. 2009;105(2):103-104. doi:10.1016/j.ijgo.2009.02.012

15. Amant F, Mirza MR, Koskas M, Creutzberg CL. Cancer of the corpus uteri. Int J Gynaecol Obstet. 2018;143(Suppl 2):37-50. doi:10.1002/ ijgo. 12612

16. Koh WJ, Abu-Rustum NR, Bean S, et al. Uterine neoplasms, version 1.2018, NCCN clinical practice guidelines in oncology. J Natl Compr Canc Netw. 2018;16(2):170-199. doi:10.6004/ jncen.2018.0006 
17. Todo Y, Okamoto K, Hayashi M, et al. A validation study of a scoring system to estimate the risk of lymph node metastasis for patients with endometrial cancer for tailoring the indication of lymphadenectomy. Gynecol Oncol. 2007;104(3):623-628. doi:10.1016/j.ygyno. 2006.10.002

18. Mitamura T, Watari H, Todo Y, et al. Lymphadenectomy can be omitted for low-risk endometrial cancer based on preoperative assessments. J Gynecol Oncol. 2014;25(4):301-305. doi:10.3802/ jgo.2014.25.4.301

19. Creasman WT, Morrow CP, Bundy BN, Homesley HD, Graham JE, Heller PB. Surgical pathologic spread patterns of endometrial cancer. A gynecologic oncology group study. Cancer. 1987;60(8 Suppl):2035-2041.

20. Milam MR, Java J, Walker JL, et al. Nodal metastasis risk in endometrioid endometrial cancer. Obstet Gynecol. 2012;119(2 Pt 1):286-292. doi:10.1097/AOG.0b013e318240de51

21. Vargas R, Rauh-Hain JA, Clemmer J, et al. Tumor size, depth of invasion, and histologic grade as prognostic factors of lymph node involvement in endometrial cancer: a SEER analysis. Gynecol Oncol. 2014;133(2):216-220. doi:10.1016/j.ygyno.2014.02.011

22. Convery PA, Cantrell LA, Di Santo N, et al. Retrospective review of an intraoperative algorithm to predict lymph node metastasis in low-grade endometrial adenocarcinoma. Gynecol Oncol. 2011;123 (1):65-70. doi:10.1016/j.ygyno.2011.06.025

23. Colombo N, Preti E, Landoni F, et al. Endometrial cancer: ESMO clinical practice guidelines for diagnosis, treatment and follow-up. Ann Oncol. 2011;22(Suppl 6):vi35-vi39. doi:10.1093/annonc/mdr374

24. Mariani A, Dowdy SC, Keeney GL, Long HJ, Lesnick TG, Podratz KC. High-risk endometrial cancer subgroups: candidates for target-based adjuvant therapy. Gynecol Oncol. 2004;95(1):120-126. doi:10.1016/j.ygyno.2004.06.042

25. Sozzi G, Uccella S, Berretta R, et al. Tumor size, an additional risk factor of local recurrence in low-risk endometrial cancer: a large multicentric retrospective study. Int J Gynecol Cancer. 2018;28 (4):684-691. doi:10.1097/IGC.0000000000001223

26. Canlorbe G, Bendifallah S, Laas E, et al. Tumor size, an additional prognostic factor to include in low-risk endometrial cancer: results of a French multicenter study. Ann Surg Oncol. 2016;23(1):171-177. doi:10.1245/s10434-015-4583-3
27. Han KH, Kim HS, Lee M, Chung HH, Song YS. Prognostic factors for tumor recurrence in endometrioid endometrial cancer stages IA and IB. Medicine (Baltimore). 2017;96(21):e6976. doi:10.1097/ MD.0000000000006976

28. Ayhan A, Sahin H, Sari ME, Yalcin I, Haberal A, Meydanli MM. Prognostic significance of lymphovascular space invasion in low-risk endometrial cancer. Int J Gynecol Cancer. 2019;29(3):505-512. doi:10.1136/ijgc-2018-000069

29. Gungorduk K, Firat Cuylan Z, Kahramanoglu I, et al. Risk factors for recurrence in low-risk endometrial cancer: a case-control study. Oncol Res Treat. 2018;41(7-8):466-470. doi:10.1159/000488112

30. Creutzberg CL, van Putten WL, Koper PC, et al. Surgery and postoperative radiotherapy versus surgery alone for patients with stage- 1 endometrial carcinoma: multicentre randomised trial. PORTEC study group. Post operative radiation therapy in endometrial carcinoma. Lancet. 2000;355(9213):1404-1411. doi:10.1016/S0140-6736(00) 02139-5

31. Bendifallah S, Canlorbe G, Huguet F, et al. A risk scoring system to determine recurrence in early-stage type 1 endometrial cancer: a French multicentre study. Ann Surg Oncol. 2014;21 (13):4239-4245. doi:10.1245/s10434-014-3864-6

32. Stephan JM, Hansen J, Samuelson M, et al. Intra-operative frozen section results reliably predict final pathology in endometrial cancer. Gynecol Oncol. 2014;133(3):499-505. doi:10.1016/j.ygyno.2014. 03.569

33. Kumar S, Medeiros F, Dowdy SC, et al. A prospective assessment of the reliability of frozen section to direct intraoperative decision making in endometrial cancer. Gynecol Oncol. 2012;127(3):525-531. doi:10.1016/j.ygyno.2012.08.024

34. Walker JL. Re: clinical practice guidelines for the management of patients with endometrial cancer in France. Int $J$ Gynecol Cancer. 2012;22(2):179-181. doi:10.1097/IGC.0b013e318231ad4a

35. Wu Y, Zhu H, Li Y. The diagnose accordance rate of endometrial cancer between intraoperative frozen section and postoperative paraffin pathology and its influencing factors. Int $J$ Obstet Gynecol. 2013;40(2):182-186.
Cancer Management and Research

\section{Publish your work in this journal}

Cancer Management and Research is an international, peer-reviewed open access journal focusing on cancer research and the optimal use of preventative and integrated treatment interventions to achieve improved outcomes, enhanced survival and quality of life for the cancer patient.
Dovepress

The manuscript management system is completely online and includes a very quick and fair peer-review system, which is all easy to use. Visit http://www.dovepress.com/testimonials.php to read real quotes from published authors. 